\title{
DOE/ER/13า70--T3
}

\section{DISTRIBUTED MEASUREMENTS OF TRACER RESPONSE ON PACKED BED FLOWS USING A FIBEROPTIC PROBE ARRAY}

Final Report under Interagency Agreement No. DE-AI05-87ER13770 Entitled:

Convection and Dispersion in Coarse-Packed Beds

Prepared for:

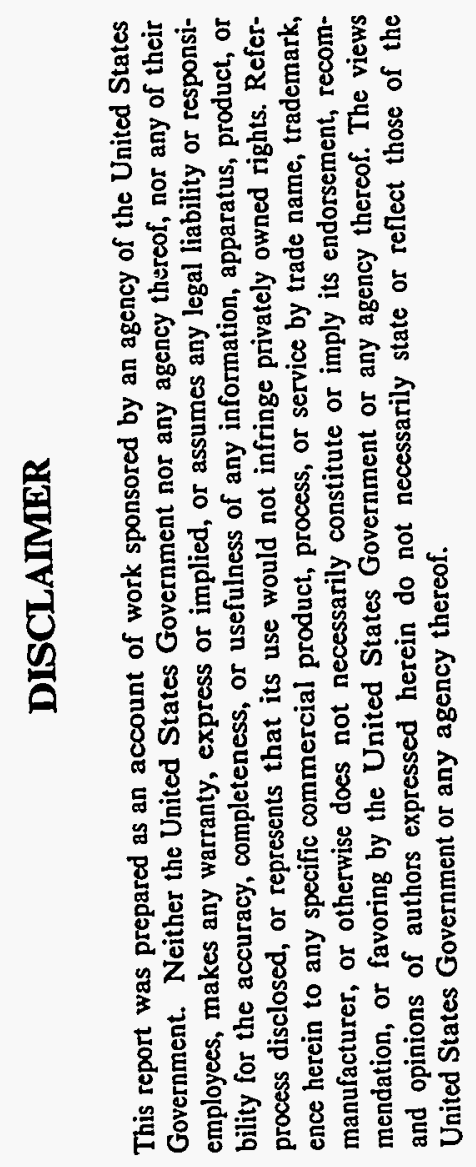

\author{
U.S. Department of Energy \\ Office of Basic Energy Sciences \\ Division of Engineering and Geosciences
}

Attention: Dr. Oscar P. Manley

Prepared by: Michael C. Jones

Chemical Science and Technology Laboratory

National Institute of Standards and Technology

Boulder, CO 80303

October 28, 1994 
'queunoop

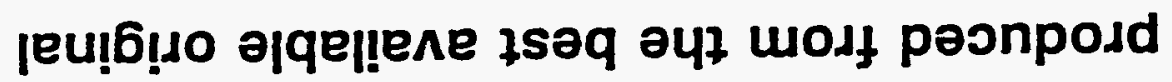

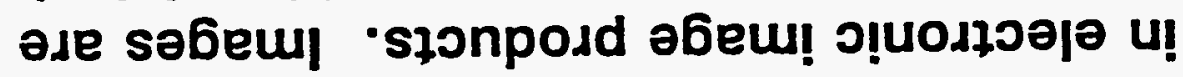

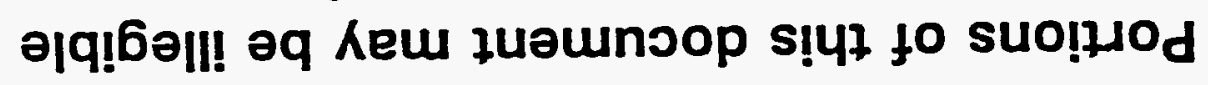

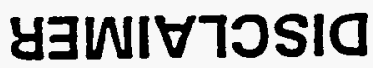




\title{
DISTRIBUTED MEASUREMENTS OF TRACER RESPONSE ON PACKED BED FLOWS USING A FIBEROPTIC PROBE ARRAY
}

\author{
Michael C. Jones*, Ryan Nassimbene ${ }^{*}$, Jonathan Wolfe $\ddagger$ \\ Chemical Science and Technology Laboratory \\ National Institute of Standards and Technology \\ Boulder, $\mathrm{CO}, 80303$ \\ and \\ Nolan Frederick \\ Rocky Mountain Electron Video \\ Boulder, CO 80303
}

\begin{abstract}
Scale-up of packed bed processes, particularly those involving chromatographic separations, is made difficult by a seemingly inevitable increase in dispersion due to packing nonuniformity. To provide a suitable chatacterization, we measured the spatial distribution of dispersion and mixing in packed beds of uniform impervious spherical glass particles by a tracer impulse technique. The key feature in our work is the use of a fiberoptic array at the exit plane to obtain a time-resolved spatially distributed response. All experiments were in the creeping flow regime. We used a fluorescent dye with laser excitation through the fiber terminations in the bed. The fluoresced radiation was collected through the same fibers. We analyzed the data by the use of indeces of the extent of micromixing based on Danckwerts's original degree of segregation and an additional index of structural uniformity. The computations involve a moment analysis of the individual and average probe responses. A simple model gives expressions for the indeces in terms of the Peclet number and is shown to provide a useful limiting case. The computed indices are also shown to be very sensitive to adsorption of dye on the surface of the glass. However, for some of the experiments with the largest spheres using Pyrex glass, the effects of adsorption are indiscernible. This technique successfully separates the contribution of micromixed fluid to overall bed dispersion from the contribution due to the transverse variation of the flow residence time.
\end{abstract}

* Current address, Colorado School of Mines, Golden, CO 80401

$\dagger$ Current address, Golden Technologies, Golden, CO 80401

$\ddagger$ Current address, Molten Metal Technology, Inc., Waltham, MA 02154 


\section{INTRODUCTION}

In industrial and laboratory applications, packed beds are most often required to effect or maintain a separation in a flowing fluid mixture. Common examples are gas adsorption, ion exchange, catalytic reactors, and chromatographic separations. In the last example, spatial separation is particularly important. Separation is promoted when all forms of axial mixing are minimized, the ideal limit being plug flow in the absence of axial dispersion and diffusional resistance. In this case, components once separated by adsorptive processes would remain separated. The ideal may be approached, particularly at the laboratory scale, by the use of very small uniform sized particles. Scaling up to process dimensions is usually achieved by increasing the bed diameter and bed uniformity becomes an issue. It is generally accepted that the degree of separation attainable will be compromised by increased dispersion which may have its origin in local variations in hydraulic permeability and manifolding problems (Gibbs and Lightfoot, 1986). It would be useful to be able to characterize a bed, not only in terms of an overall dispersion coefficient, but also in terms of the contribution from packing nonuniformity transverse to the flow direction, since this has at least the potential to be eliminated.

Our interest in this area was motivated by this and by the development in our laboratory of sensitive fiberoptic probes which presented the opportunity of obtaining information on the distributed performance of a packed bed. The probes are used to excite and detect fluorescence of a tracer dye which produces a signal proportional to dye concentration. The ends of a number of fibers may be inserted at a plane just upstream of the bed outlet in such a way that the packing upstream is undisturbed. We thus obtain in situ measurements of concentration history distributed across an exit plane in response to tracer dye injection. They are in situ in the sense that the measurements are made before the final mixing step where the effluent is brought together in a single stream. This technique provides a great increase in the amount of information available over conventional tracer injection experiments. The challenge is to use this information to provide useful characterization of the packed bed and the fluid transport processes.

In this paper, we illustrate some approaches to the utilization of this distributed response. We employ the concepts-of micromixed and macroscopically mixed fluid introduced by Danckwerts (1958) and by Zwietering (1959) to define criteria applicable to axial flow geometry in packed beds. We compare the results with theoretical calculations based on simple models and we find that the statistics of the multiple probe responses do indeed describe micromixing in an illuminating way.

To narrow the scope, we used packings of nonporous glass spheres. Initially, we had supposed that the small surface area available for adsorption of tracer dye on the external surface of the glass spheres would mitigate any effect on the hydrodynamics of dispersion, but we find that even in the case of the largest spheres studied ( $3 \mathrm{~mm}$ diameter) the effect of adsorption on the tracer response must be considered. While the smallest diameter studied $(0.275 \mathrm{~mm})$ is still a factor of five larger than finds use in typical large scale 
chromatography, we feel that we are now in a position to study these smaller sizes based on what has been learned here.

\section{APPLICATION OF MIXING CRITERIA TO PACKED BEDS}

The concept of a "point" in a chemical reactor was introduced by Danckwerts as a differential volume -- small compared with the reactor fluid volume but large enough to contain a large number of molecules with a distribution of ages. This permitted the definition of the degree of segregation:

$$
J_{s}=\frac{\text { variance of mean ages between points }}{\text { variance of ages over entire volume }} \quad \begin{array}{r}
\operatorname{var} \alpha \\
\hdashline-\alpha_{p}
\end{array}
$$

Zwietering showed that

$\operatorname{var} \alpha=\operatorname{var} \alpha_{p}+(I / /) \iiint_{V}\left(\sigma_{p}\right)^{2} d V$

in which the integral sums the variances of ages within points, the variances being given by:

$$
\sigma_{p}^{2}=\int_{0}^{\infty}\left(\alpha-\alpha_{p}\right)^{2} \phi_{p}(\alpha) d \alpha
$$

Here, $\phi_{p}(\alpha)$ is the local age probability density function. The second term on the right hand side in (2) is the contribution of micromixing to var $\alpha$. When it is zero at a point, the fluid is said to be locally segregated; when it is zero for all points, $J_{S}=1$. Certainly, in separations, a high degree of segregation is desired and a knowledge of $J_{S}$ would be a useful measure.

Unfortunately, although a powerful concept, this index is, generally, not directly measurable because of the need for instantaneous measurement throughout the entire fluid volume in order to calculate $\alpha_{p}$ and its variance. On the other hand, the denominator in (1) -- the variance of the fluid ages for the entire population of molecules in the vessel -can be found from the residence time distribution. Our experimental approach offers a partial solution to the problem by placing a number of tracer probes on a plane perpendicular to the main axis of flow at the bed exit plane. The experimental details are discussed below. Each probe supplies age information for part of the bed upstream of it. If we could trace molecules back upstream from a point on this plane, we would trace out a "filament" of flow originating at the inlet plane. Such filaments would possibly branch so that a point on the probe plane might originate from several points on the inlet plane. The entire fluid volume is accounted for, presumably, by the filaments emanating from a sufficient number of sampling points. We replace the differential volume of Danckwerts's "points" by "filaments", possibly branched, and define a new index of segregation exactly parallel to his degree of segregation: 


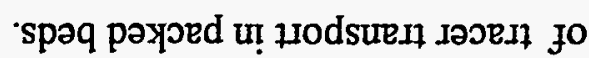

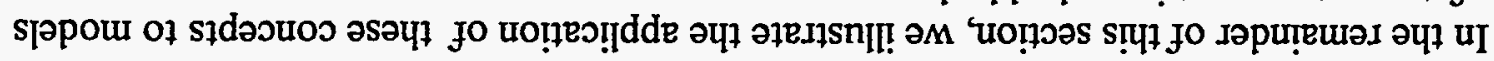

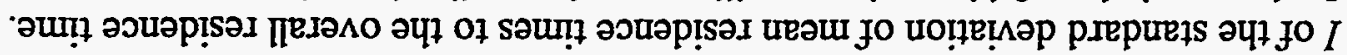

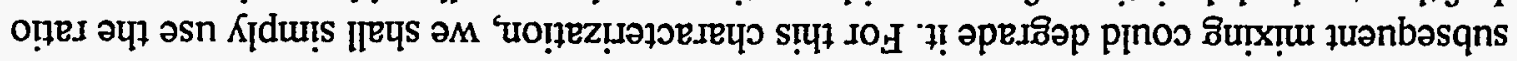

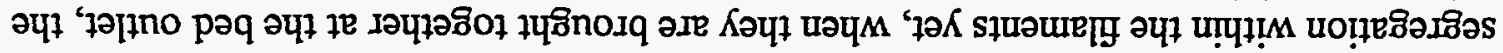

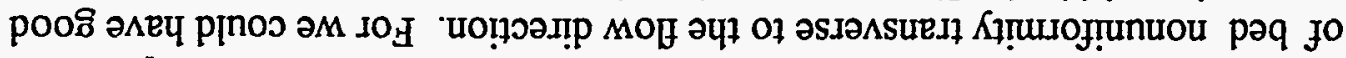

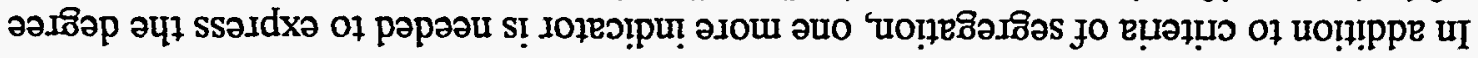

MOF

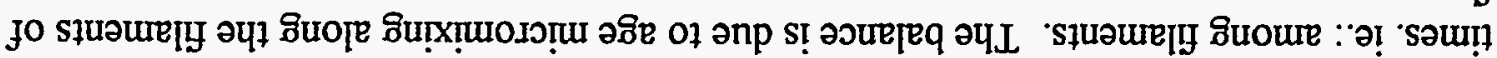

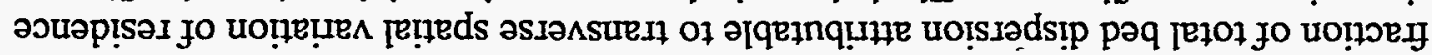

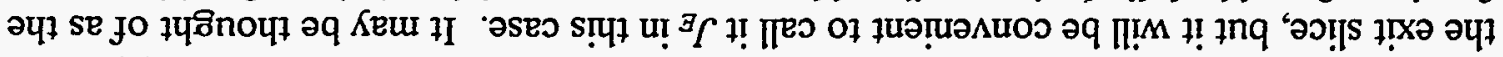

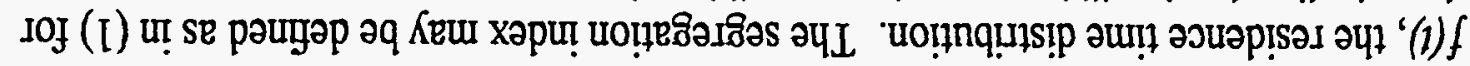

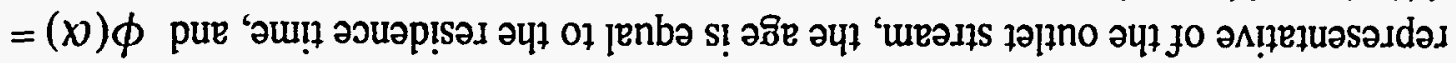

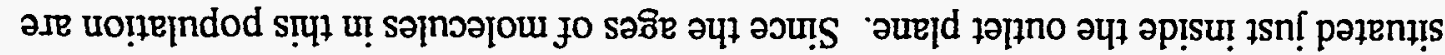

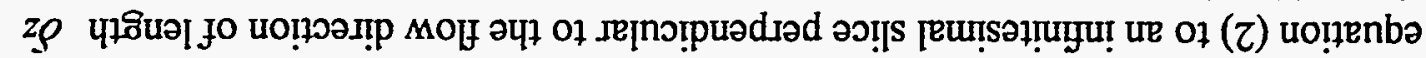

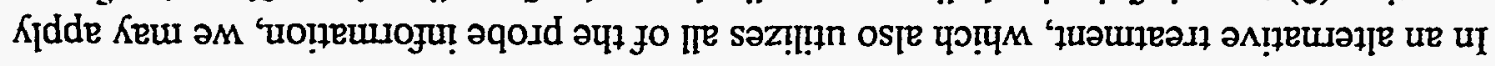

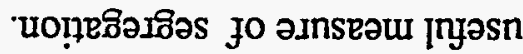

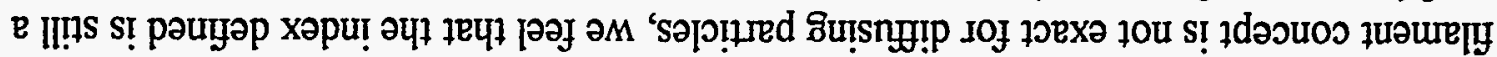

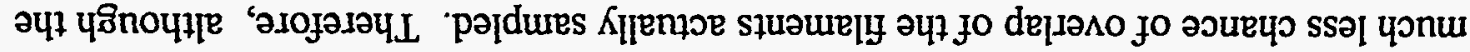

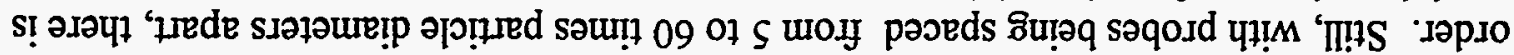

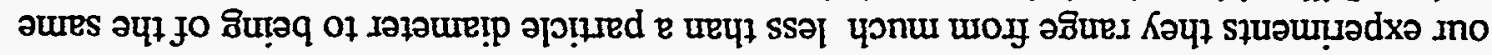

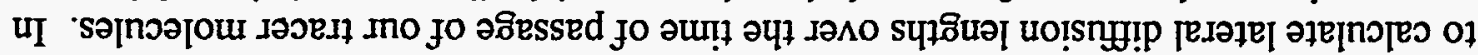

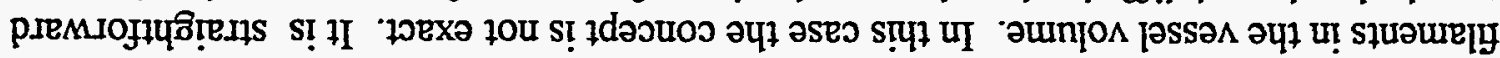

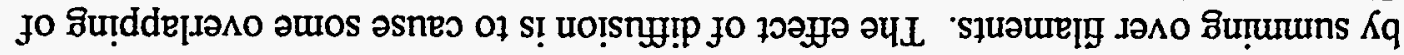

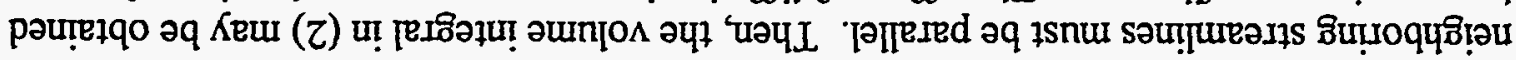

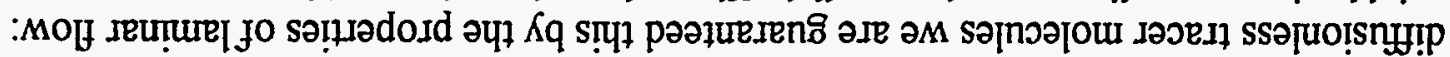

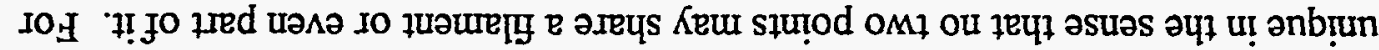

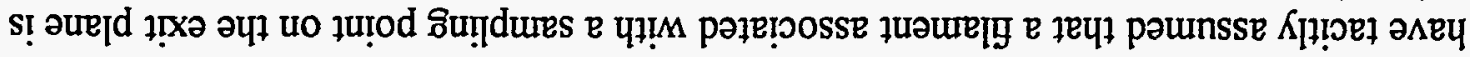

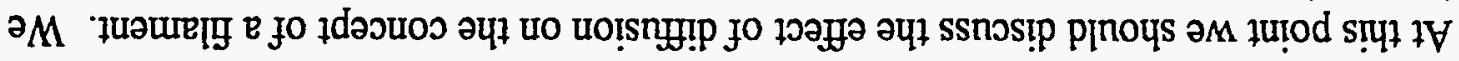

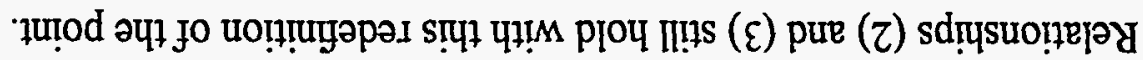

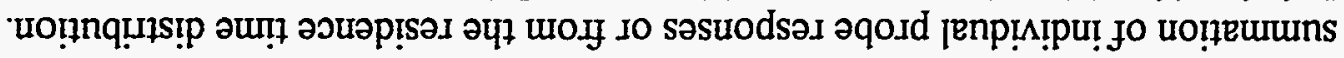

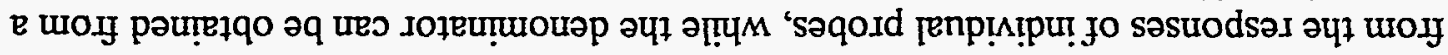

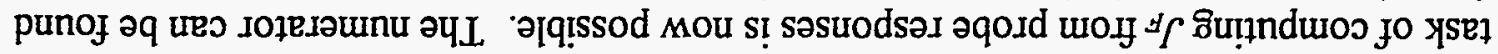

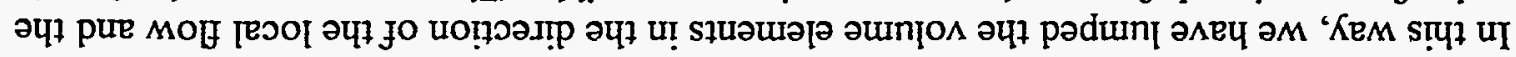

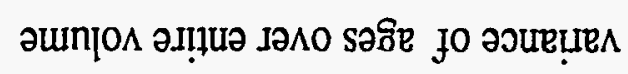

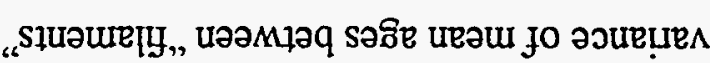


In this most common model of packed bed flow, we observe that by calculating a onedimensional, axial flow case we will have lumped the transverse concentration distribution and we cannot calculate $J_{F}$ or $J_{E}$. A calculation of $J_{S}$ will only take into account concentration distributions in the axial direction. But the derivation illustrates the application of age distribution methods and we obtain other useful results.

One version of this model includes the possibility of adsorption of tracer dye on the surface of the particles. We write the differential equation for the concentration $C$ of a given specie in the interstitial fluid as follows:

$\frac{\partial C}{\partial}+v \frac{\partial C}{\partial z}=E \frac{\partial^{2} C}{\partial z^{2}}+\frac{n a}{\varepsilon}$

Here, $v$ is the interstitial velocity, $E$ the dispersion coefficient, $a$ the specific external area of the packing and $n$ is the mass flux of adsorbed specie into the interstitial fluid. A hierarchy of models exists for $n$ depending on the nature of the packing. To match our experiments, we limit our scope here to uniform impermeable spherical particles on which adsorption may occur. If $C_{a}$ is the adsorbed concentration per unit surface area, its rate of change may be described by

$\frac{\partial C_{a}}{\partial t}=k_{a}\left(C_{i}-C_{a} / K\right)=k_{m}\left(C-C_{i}\right)=-n$

In this equation, $C_{i}$ is the concentration in the fluid at the solid-liquid interface, $k_{a}$ the adsorption rate constant, $k_{m}$ the external fluid-mechanical mass transfer coefficient and $K$ is the equilibrium constant. If we define $C^{*}=C_{d} / K$, we may rewrite (6) as

$K \frac{\partial C^{*}}{\partial t}=k_{o f}\left(C-C^{*}\right)=-n$

Where $k_{e f f}=\left(1 / k_{a}+1 / k_{m}\right)^{-1}$. We may set equations (5) and (7) in nondimensional form choosing $L$ for the length scale and $L / v$ for the time scale. With $z$ and $t$ thus redefined,

$\frac{\partial C}{\partial t}+\frac{\partial C}{\partial z}=\frac{1}{P e} \frac{\partial^{2} C}{\partial z^{2}}+\frac{a L}{\varepsilon v} n$

with $P e \equiv v L / E$, and

$K \frac{\partial C^{*}}{\partial t}=k_{g f}\left(C-C^{*}\right)=-\frac{L}{v} n$

Nauman and Buffham (1983) give a useful discussion of the relationship between the impulse response function for open boundaries and the residence time distribution. Suffice it to say here that, for large $P e$, the residence time distribution (closed system boundary 
conditions) is identical to the more straightforward impulse response function (open system with concentration continuous across bed inlet boundary and bounded at infinity) which we calculate here. Practically, we require $P_{e}>16$, which is satisfied in all our experiments. The appropriate initial and boundary conditions for unit impulse injection of a tracer at $z=0$ are:

$C(z, 0)=0$

$C(0, t)=\delta(t)$

$C(\infty, t)=$ bounded

When we take Laplace transforms of equations (8),(9) and (10) with respect to $t$, eliminate $n$ and $C^{*}$ and solve the resulting ordinary differential equation in $z$ for $C(z, s)$, we get the following solution in the Laplace domain:

$C(z, s)=\exp [(P e / 2)(z)(1-\sqrt{ }(I+4 G(s)(z), P e))]$

in which $s$ is the Laplace transform variable, and

$G(s)=[s+P(I-I /(s / Q+1))]$

$P \equiv a k_{e f f} L / \varepsilon v$ and $Q \equiv k_{e f f} L / K v$. We shall also use the ratio $R \equiv P / Q=a K / \varepsilon$. This is a special form of the capacity factor familiar in chromatography and it represents the equilibrium ratio of tracer mass on the surface of the spheres (stationary phase) to that dissolved in the fluid (mobile phase) for a unit of bed volume.

Inversion of $(11)$ in the general case, where mass transfer rates are important, is beyond the scope of the current work. But, in the limit $Q=\infty$, inversion of equation (11) with (12) is straightforward. This is the limit of equilibrium adsorption. For this case, $G(s)=$ $s(I+R)$ and we find for the time domain solution:

$C(z, t)=\left((P e . z(I+R)) / 4 \pi t^{3}\right)^{1 / 2} \exp \{-[P e . z(t(1+R) t)][t-(l+R) / 2\}$

Moments of the general case may be obtained from (11). Here we state the moments only for equation (13), but we give ratios of general moments to these equilibrium adsorption moments in an appendix. We take $z=1$.

$\mu_{0}=I$

$\mu_{1}=(1+R)$

$\mu_{2}=(1+2 / P e)(1+R)^{2}$

$\mu_{3}=\left(1+6 / P e+12 / P e^{2}\right)(I+R)^{3}$

In view of (14), the function in (13) may be regarded as the residence time probability density function (or, more simply, the residence time distribution) which we shall also designate as $f(t)$ when $z=\ell$. It is easy to show that solutions for all values of $R$ are self 
similar in time units of $L^{\prime}(v(l+R))$. The effect of equilibrium adsorption is simply to retard and broaden the transport of tracer in real time. A consequence of this self similarity is that in fitting experimental data the mean residence time and the quantity $(I+R)$ cannot be independently determined; only their ratio can be found.

Exact relationships (eg. Nauman and Buffham (1983)) for the mean and variance of the internal age distribution, $\phi(\alpha)$, in terms of the moments of the residence time distribution are now used:

$\langle\alpha\rangle=\mu_{2} 2 \mu_{I}$

$\operatorname{var} \alpha=\mu_{3} / 3 \mu_{1}-\left(\mu_{2} / 2 \mu_{1}\right)^{2}$

hence:

$$
\begin{aligned}
& \langle\alpha\rangle=(1 / 2-1 / P e)(1+R) \\
& \operatorname{var} \alpha=\left(1 / 12+1 / P e+3 / P e^{2}\right)(I+R)^{2}
\end{aligned}
$$

Using these results, we calculate var $\alpha_{p}$ for points consisting of infinitesimal slices transverse to the $\mathrm{z}$ axis:

$$
\begin{aligned}
\text { var } \alpha_{p} & =(I / V) \iiint_{(\alpha p-\langle\alpha\rangle)^{2} d V=(I / L)} \int_{0}^{L}[z / v-(I / 2+1 / P e)(1+R)]^{2} d z \\
& =\left(1 / 12+(1 / P e)^{2}\right)^{2}(1+R)^{2}
\end{aligned}
$$

From (19A) and (21) we calculate Danckwerts's degree of segregation:

$J_{S}=\left(1 / 12+(1 / P e)^{2}\right) /\left(1 / 12+1 / P e+3 / P e^{2}\right)$

As to be expected, $J_{S}$ approaches the complete segregation limit of 1 as $P e$ approaches infinity.

This model is the basis for analysis of packed beds when only residence time distribution for the mixed outlet flow is available and all transverse variations are lumped. A practical situation it represents exactly is the packed bed consisting of a large number of parallel, identical hollow fibers suggested by Gibbs and Lightfoot (1986) for large scale chromatographic separations.

\section{B. Ideal dispersion model}

This model adds structure to the previous one by considering all axial dispersion to arise from a distribution of residence times among flow filaments -- an extreme case. Within each filament, we assume plug flow with equilibrium adsorption but no dispersion. The flow within each filament therefore remains segregated, but each transverse slice contains a distribution of ages as before. This model was developed in a quest for an upper limit to the factor $J_{F}$ for a given $f(t)$. Obviously, it could be embellished further by adding Taylor- 
Aris type dispersion within the filaments, but not without introducing detailed modeling for the filaments and additional parameters.

We first calculate the numerator of $J_{F}$. We use the subscript $i$ to denote an individual filament.

$\operatorname{var} \alpha_{p}=\sum_{i}\left(V_{i} N\right)\left[\alpha_{p,}-\langle\alpha\rangle\right]^{2}$

If we assume that a filament residence time is proportional to its volume, then $V_{1}=t_{i} Q / N$, where $Q$ is the total volumetric flow rate $N$ is the number of filaments. Substituting for $V$, we get

$\operatorname{var} \alpha_{p}=(Q / N N) \sum_{i} t_{l}\left[\alpha_{p i}-\langle\alpha\rangle\right]^{2}$

We now impose the condition that filament residence times occur with a probability distribution given by (13) in order to conform to the previous model. Then (24) can be written as

$$
\text { var } \begin{aligned}
\alpha_{p} & =(Q / V) \int_{0}^{\infty} f(t) t\left[\alpha_{p}(t)-\langle\alpha\rangle\right]^{2} d t=(Q / V) \int_{0}^{\infty} f(t) t[(t / 2)-\langle\alpha\rangle]^{2} d t \\
& =(Q / V)\left[\mu_{3} / 4-\langle\alpha\rangle \mu_{2}+\langle\alpha\rangle^{2} \mu_{1}\right]
\end{aligned}
$$

Substituting for the moments from (15)-(17) and for the mean age from (18A), and noting that $Q / V=I / \mu_{1}=1 /(1+R)$, we find

$\operatorname{var} \alpha_{p}=\left[2 / P e^{2}+1 /(2 P e)\right][1+R]^{2}$

Finally, we compute $J_{F}$ using (26) and the result for var $\alpha$ obtained in (19A):

$J_{F}=\left[2 / P e^{2}+1 /(2 P e)\right] /\left[1 / 12+1 / P e+3 / P e^{2}\right]$

It can be verified that this model satisfies equation (2) by calculating the volume integral, again assuming filament residence times are distributed according to (13).

In comparing experimental data below with (27), we have found it more convenient to plot the quantity $M_{F} \equiv\left(1 / J_{F}\right)-1$ which represents the variance of ages within filaments divided by the variance of mean ages between them. On this plot, $M_{F}(\mathrm{Pe})$ according to equation (27) is practically a straight line which is expected to be a lower limit.

It can be seen from equation (2) that the index $J_{E}$ attains the value 1 in this model since the variance of ages within the outlet slice points is zero. Furthermore, there is no dependence on $P e$. 
We can also calculate a limiting value for the index of nonuniformity, $I$. It is simply

$I=\left(\mu_{2}-\mu_{1}^{2}\right)^{12 /} / \mu_{1}=(2 / P e)^{1 / 2}$

\section{EXPERIMENTAL MEASUREMENTS}

Tracer Pulse Response

We performed tracer response experiments on vertical beds of washed uniform glass spheres in an acrylic cylindrical vessel designed to give a uniform flow distribution of water at the top and uniform outlet at the bottom corresponding as closely as possible with the models described above. Inlet flow passes through a low-volume cavity behind a $2 \mu \mathrm{m}$ stainless steel frit to ensure even distribution. We inject a fluorescent dye, Rhodamine 6G, into the inlet stream of deionized water using a conventional lowpressure, liquid-chromatography, 6-port injection valve fitted with a $5 \mathrm{ml}$ sample loop. The support plate for the bed is another stainless steel frit drilled with an array of holes to permit the introduction of an array of 61 optical fibers on a hexagonal grid, each protected by a loose fitting stainless steel tube. Each fiber is $16 \mathrm{~mm}$ from its nearest neighbor on the support plate. Both upper and lower heads of the containing vessel are supplied with cooling water under temperature control. All experiments described here were performed at $25^{\circ} \mathrm{C}$. The experimental system is shown in Figure 1.

The fiberoptic probe system in its original form was described by Perkins and Jones (1989). In the experiments described here, we use an improved design which is less expensive to construct and electronically more stable, but is similar in principle. A single probe is a pair of optical fibers, with one end of each inserted into a detector housing of machinable plastic. We use polymer-clad silica fibers of $0.23 \mathrm{~mm}$ overall diameter and $0.20 \mathrm{~mm}$ core diameter. One of the remaining ends receives light from a $488 \mathrm{~nm}$ argon ion laser as part of a bundle of such fibers in the laser beam, while the other end is inserted into the packed bed. All fiber ends are carefully cleaved to ensure efficient coupling of light into and out of the fibers. The path of the $488 \mathrm{~nm}$ excitation light from the laser is first to the detector housing where it is reflected back into its pair by a high-pass filter deposited on the far end of a collimating gradient index (GRIN) lens. On entering the fluid-filled packed bed, a fraction of the $590 \mathrm{~nm}$ fluorescent light emitted from any dye present returns along the same fiber back to the detector housing where it now passes the GRIN lens and high-pass filter. It then passes a $30 \mathrm{~nm}$ band-pass filter centered at 590 $\mathrm{nm}$, and, finally, impinges on a silicon photodiode detector. Signals from the photodiodes are amplified by individual electrometer grade operational amplifiers with "Tee" feedback networks of $10^{10} \Omega$ equivalent resistance. Outputs are processed by 14 bit analog-to-digital converters and recorded on a personal computer. Typical responses are in the range 0.1 to $0.3 \mathrm{~V} / \mathrm{ppm}$ of dye with a high degree of linearity down to 0.1 ppm; peak concentrations are from 20 to $100 \mathrm{ppm}$ for $1000 \mathrm{ppm}$ injection.

We have determined that these optical fiber probes sample a small volume within a few fiber diameters of the fiber end. 
The glass spheres used were $3 \mathrm{~mm}$ dia. Pyrex; $1.5 \mathrm{~mm}$ dia. industrial grade soda lime glass; and $0.275 \mathrm{~mm}$ dia. lead free soda lime. We tried to eliminate variability of packing density as a factor in the results by using the highest density possible. It is well known that the method of introducing the glass spheres into the containing vessel is quite important here and we found the highest density when thoroughly dry spheres were poured from such a height that a good degree of bouncing was obtained. For 1.5 and $3.0 \mathrm{~mm}$ spheres, the void volume of each bed could be measured in situ by displacing air from below with water to a known height. Capillary rise due to surface tension made this impossible with the $0.275 \mathrm{~mm}$ spheres, therefore, for these spheres, we used a method of adding typical dry sphere beds of known bulk volume to known volumes of water and measuring displaced volume. Bulk mean void fractions of $0.376,0.367$ and 0.367 were measured respectively for the $0.275,1.5$ and $3.0 \mathrm{~mm}$ spheres. This compares with best values reported for random beds of uniform spheres of 0.36 reported by Haughy and Beveridge (1966).

The experimental procedure is straightforward. After packing the bed with washed, dried spheres and measuring its void volume, we start reverse flow of deionized water through the bed by gravity feed displacing air upward through from the bed and the distributor cavity. When complete, forward flow of deaerated deionized water from a reservoir is established via a variable speed peristaltic pump. Calibration of the fiberoptic probes is performed after several hours of steady flow by switching to a second reservoir containing $20 \mathrm{ppm}$ of dye. We establish zero and slope knowing the response to be linear. A $5 \mathrm{ml}$ injection of $1000 \mathrm{ppm}$ dye solution starts a run when, again, pure water has been pumped through the bed for several hours at the chosen flow rate. The probe signals are read at intervals of from 5 to $25 \mathrm{~s}$ chosen to provide usually 500 data points for each probe for each injection event or, run. All 61 probes can be read within $11 \mathrm{~ms}$. Run times from 2,500 to $12,500 \mathrm{~s}$, depending on flow rate, are chosen to give as much of the tail of a response as possible. Data files of concentration vs. time are created and transferred to diskettes for later processing along with other run information such as flow rate, temperature, particle diameter and bed depth. Figure 2 shows a typical plot of the complete concentration/time data set from one run.

\section{Adsorption Measurements}

After it became evident that a second delayed component was present in probe responses, possibly due to adsorption/desorption of dye on the surface of the glass spheres, we made screening tests of adsorption in separate experiments. We equilibrated samples of the glass spheres with solutions of rhodamine- $6 \mathrm{G}$ in deionized water at known concentrations. Enough solution was used to cover 100 to $200 \mathrm{~g}$ of spheres in a conical flask. Frequent agitation was applied over a period of at least 24 hours. Solutions of 50,100 and 500 ppm were used. We then obtained concentrations before and after contact by measurements of absorbance at $400 \mathrm{~nm}$ using a diode array spectrometer. The fall in concentration was easily measured. Equilibrium loadings from $0.1(10)^{-3} \mathrm{~g} / \mathrm{m}^{2}$ to $3.5(10)^{-3}$ $\mathrm{g} / \mathrm{m}^{2}$ were found for all three sphere sizes. These results compare well with the value of $2.25(10)^{-3} \mathrm{~g} / \mathrm{m}^{2}$ calculated from published $\mathrm{OH}$ surface concentrations for hydroxylated glass given by Lee et al. (1984) which, along with heavy metal impurities are thought to be 
the sites for adsorption of molecules with zones of high electron concentration. We also noticed that, while the $3 \mathrm{~mm}$ (Pyrex glass) beads could be easily rinsed of residual dye after 2 or 3 washings in deionized water, the 1.5 and $0.275 \mathrm{~mm}$ (soda-lime glass) spheres and the rinse water retained coloration after 20 to 30 washes, an observation in accord with some of our findings on tailing discussed below.

From these loadings, we calculate capacity factors $R$ on the assumption that they represent linear adsorption. There is evidence, however, of approach to saturation so we used largest values in order to asses the degree of retardation that could be expected from adsorption. For the 3, 1.5, and $0.275 \mathrm{~mm}$ spheres respectively, we find capacity factors of $0.13,0.65$, and 1.36 . These are certainly large enough for us to observe retardation if equilibrium adsorption is the applicable mechanism.

\section{DATA REDUCTION AND RESULTS}

Tracer response data reduction takes place in two phases. We first characterize the overall bed response in terms of equation (13) and determine the zeroth through third moments to calculate var $\alpha$ via equation (19). Then we need the individual probe statistics. We find first and second moments of individual probe responses in order to calculate $\alpha_{p}$ for the filament associated with each probe (equation (18) applied to an individual filament). From this follows the mean of the $\alpha_{p}$ 's and their variance, var $\alpha_{p}$, thus allowing us to calculate $J_{F}$. A similar procedure applied to the probe residence times enables us to calculate $J_{E}$.

\section{Overall bed response}

We compute the overall response by averaging the concentrations recorded for the probes at each time step. A practical problem that must first be dealt with is that, for an individual run, there may be a number of casualties among the 61 probes. The causes may be of electronic or optical origin. Of the latter, we have found the following to be possible causes: chipped fiber end; fiber end placement relative to spheres; fiber attachment in detector module becoming strained; poor illumination in the laser beam. Rareiy were there problems with the electronics once the circuits were debugged. This loss of some probes raises the important question of redundancy: how many probes are needed? We answered this question as follows.

Neglecting for now wall effects, each probe may be considered to sample a part of the flow through a random arrangement of spheres in the packing. Provided the probes are more than 3 or 4 particle diameters apart, we can be confident that the flow seen by one probe is an independent sample of the flow. (Even in the worst case, with $3 \mathrm{~mm}$ spheres, we have more than 5 diameters separation. ) Then the question may be rephrased: how many of such independent samples are required to give moments of the probe-mean response that are statistically stationary? Figure 3 shows a typical plot of the second moment calculated from $2,3,4 \ldots . . . N$ probes. In selecting probes to average in this figure, we chose them at random to eliminate any sequence with spatial ordering. From this plot it is clear that anything over 30 will give consistent results. This is a comfortable margin of safety, but first it is necessary to decide on criteria for the rejection of a probe based on 
its response, and here some judgment was necessary. We rejected probes where there was excessive noise, zero response, or excessive drift. Most individual probe responses had both noise levels and drift much less than $1 \mathrm{ppm}$. We rejected a probe when either was greater than 2 ppm or when peak response was less than $5 \mathrm{ppm}$. With these criteria, we usually had more than 50 good probes. In the worst case we had 39.

We tried fitting the average probe response to equation (13). The fits were usually not good; we could not obtain good fits to the tails of the response. Equation (13) is not capable of accurately reproducing the experimentally observed shape. This is often assumed in the literature to be caused by diffusion effects and parasitic external mixing volumes (Hejtmanek and Schneider, 1993). In our case, downstream volumes can be eliminated from consideration since the probes are actually in direct contact with the packing. Furthermore, our injection volumes and distributor volumes are each less than 1 $\%$ of the liquid volume of the packing and are not considered significant.

Excellent fits to the data resulted when we used a linear combination of two such functions as equation (13) as a model equation. This is illustrated in Figure 4. In almost all cases, the overall response can be decomposed into a main component plus a smaller delayed broader component. In many cases the second component manifests itself as an obvious shoulder while, in one case, the shoulder resolved into a second broad maximum. This behavior is seen not only in the overall response but also in individual probe responses.

The curve-fit functions facilitate the computation of first second and third moments of the overall system response by numerical integration. This is particularly important for the third moment where we minimize truncation errors by extrapolation to much larger times than are practical in experiments. From each fit we also get the parameters mean time and $P e$ for each of the two components and the ratio of their areas.

\section{Individual probe statistics}

In equation (18) above, the mean age for the entire system is obtained in terms of first and second moments of the residence time distribution. Since each probe records the response of a distinct part of the flow -- a filament of the flow -- the same equation applied to probe response gives the mean age for its filament. We obtain probe first and second moments by straightforward numerical integration. Then the variance of the probe mean ages over the population of all functioning probes yields var $\alpha_{p}$ for use in calculating $J_{F}$. Some truncation error is incurred.

The calculation of $J_{E}$ is more straightforward; we need only the variance of the mean residence times over probes, var $t_{p}$, to calculate the numerator of (1).

\section{Errors}

We have to be content with a range of uncertainty for the value of $P e$ obtained from the average probe response for a particular run: the lower value from a fit of equation (13) 
and an upper value when we use a linear combination of two equations (13) each with its own parameters. We assume in this case that the early major component characterizes the hydrodynamic conditions of the flow and provides the parameter $P e$. The range is shown as an error bar in Figures 5 and 9 , which are plots of $M_{F}$ vs $P e$ and $P e_{d}$ vs $R e$ respectively and where the mean of the range is plotted. The $95 \%$ confidence intervals on the values of $P e$ from the curve fits are in the range of 2 to $9 \%$ and are much less than the above range of uncertainty. We estimate an upper bound to the truncation errors in first and second moments for a given probe from numerical integrations of the response function, equation (13), to selected truncation times, and comparison with integration to virtual infinity. For the majority of runs, the error in the quantity $M_{F}$ due to truncation is only a few percent; the error bars are of approximately the same size as the symbol plotted and are therefore not shown. A more serious problem arises in the case of the $0.275 \mathrm{~mm}$ spheres where tailing is particularly severe, and long run times were necessary. In this case we find very large contributions to $\mu_{2}$ and $\mu_{3}$ from the tails. These values are subject to the uncertainties of instrument drift and appear to be devoid of physical meaning. Therefore, values of $M_{F}$ are not reported for those runs. This is not to say that the tails themselves are devoid of physical meaning. We believe the tails are real and indicative of a very slow desorption, precisely as observed in our adsorption measurements when we found excessive washing necessary to completely remove the dye.

\section{DISCUSSION}

When we compare the mean time $(L / v)$ parameter obtained from the fits to overall responses (parameter a in Figure 4) with the known experimental value from $\epsilon V_{d} / Q$, we find no evidence of retardation due to equilibrium adsorption for any of the particle sizes; all $L / \nu$ parameters are within a few percent of the experimental values. This is important for it enables us to identify the main component of the response as being of purely hydrodynamic origin. It represents tracer that was not held up by adsorption and indicates that either adsorption must be a slow process on the scale of the residence time or that saturation is quickly reached so that most tracer molecules have no opportunity for adsorption. We tend to favor the first possibility in view of the tailing phenomenon.

In Figure 5, we obtain new insights into the measurements. The quantity $M_{F}=1 / J_{F}-1$ may be thought of as the ratio of dispersion at the micromixing level (within a filament) to dispersion among the filaments. The ideal'dispersion model represented by the solid line does indeed seem to provide a lower limit. But many runs show large values, the $1.5 \mathrm{~mm}$ spheres of bed 7 showing a declining trend with $P e$. The contrast between the $3 \mathrm{~mm}$ bed 8 and the $1.5 \mathrm{~mm}$ bed is even more striking when we plot $M_{F} v s R e$ in Figure 6 along with best fit curves through the respective sets. It appears that the excessive micromixing is flow dependent and we suspect that we are seeing rate-limited adsorption/desorption in the $1.5 \mathrm{~mm}$ bed. On the contrary, with the $3 \mathrm{~mm}$ bed there is absolutely no effect of flow rate.

Figure 7 shows the plot of $M_{E}=1 / J_{E}-1$ which applies strictly to the exit plane and may be arrived at directly from probe responses without the necessity to calculate the higher moments. We recall that in the ideal dispersion model the fluid in a filament remains 
segregated and therefor $J_{E}=1 ; M_{E}=0$. Once again, excessive micromixing is characteristic of the smaller particles while the lowest values of $M_{E}$ belong to bed 5 . It is significant that the fit to the responses of bed 5 show no delay in the second component and the fits are excellent throughout the range: the tailing phenomenon is absent. The contrast between $3 \mathrm{~mm}$ particles of beds 5 and 8 in the case of $M_{F}$ is not as clear as for $M_{E}$ and make $M_{E}$ perhaps the more useful index of micromixing.

The index of nonuniformity $I$ is shown in Figure 8. The index lies within the range 0.05 to 0.13 for all beds and is about the same for them all. The ideal dispersion model is a good upper limit.

Finally, in Figure 9 we show a conventional correlation of dispersion through the particle Peclet number, $P e_{d}$, plotted against particle Reynolds number $R e$. This is a plot suggested by hydrodynamics and dimensional analysis. Older results show a generally constant value for $P e_{d}$ and are generally lower, a good representation being the correlation of Wen and Fan (1975). It gives a practically constant value of 0.556 for $P e_{d}$ in our range of $R e$. Hejtmanek and Schneider (1993) proposed a more general correlation which would take into account the effect of molecular diffusion as a mechanism in dispersion through the Schmidt number, $S c$, which they varied over a wide range in their experiments.

$P e_{d}=0.863(\operatorname{ReSc})^{-0.078}, \quad 1<\operatorname{ReSc}<1500$

We show this correlation for Rhodamine- $6 \mathrm{G}$ for which we estimated a diffusion coefficient of $4.0(10)^{-10} \mathrm{~m}^{2} / \mathrm{s}$ and a Schmidt No. of 2250 by the Wilke-Chang correlation and the method outlined in Reid et al. (1986). While equation (28) gives values significantly higher than older results, our results are even higher. Moreover, our interpretation of the role of adsorption would lead us to favor the upper extent of the error bars. On the other hand, the decline in $P e_{d}$ shown by equation (28) is supported by our results. It is difficult to find reasons why dispersion should be less in our experiments than in previous work. While it is true that we have eliminated some of the causes of parasitic dispersion by making in situ measurements, the difference is rather striking. A factor that may possibly play a role is that dispersion is usually obtained from the "cup mixed" concentration of the outlet stream of a vessel. In our case, we have used the expectation value of the tracer concentration at a given time over the probe population. We have no way of estimating the consequence at present and this question remains open for further clarification.

These measurements, distributed over the exit plane, have provided a means of separating dispersion into two components: that due to micromixing in a flow filament and that due to variation among filaments. Adsorption gives a false indication for dispersion as it is species specific and does not represent the hydrodynamic behavior of the fluid. Among results for which adsorption may be eliminated (and this would not be the case for most dye-glass combinations) it is not yet possible to distinguish between dispersion from the Taylor-Aris mechanism ( boundary-layer holdup) on the one hand, and from a contribution which must arise if a filament is formed by the confluence of several streams 
originating from different points on the inlet plane. Such streams may have different ages at the point of confluence. However, in creeping flow, streamlines cannot converge and this may only happen by the mechanism of diffusion transverse to the flow direction. It would be useful to perform a future experiment with a large nondiffusing molecule to further clarify these two contributions to dispersion.

\section{CONCLUSIONS}

Being able to simultaneously observe tracer response at points distributed over the exit plane leads to insights into the operation of a fixed bed. Analysis in terms of indices of segregation applied to filaments of flow allow an understanding in terms of micromixing. The ideal dispersion model provides a useful limiting case and a lower bound on the possible value of $M_{F}$ as a function of $P e$, where $M_{F}$ represents the ratio of age dispersion due to micromixing to that from transverse variation of ages. Adsorption of tracer dye, somewhat unexpected at the outset, but which we confirmed by separate measurements, gives rise to excessive micromixing for the tracer. This is a false indication for the fluid, and is revealed when $M_{F}$ is plotted against $R e$. The interpretation is reinforced when the index $M_{E}$ is examined. For this index, the closest approach to the ideal dispersion model occurred precisely for those beds for which we saw the least distortion from the dispersion shape, equation (13). Our data for bed 5 are believed to be essentially free of adsorption effects. The gross uniformity of our beds transverse to the flow direction was good as indicated first by the low values of overall void fraction and also by the index of nonuniformity, $I$.

\section{ACKNOWLEDGMENTS}

This work was performed under DoE contract DE-A105-87ER13770 and we thank Dr. Oscar P. Manley for his continued support and encouragement.

\section{NOTATION}

a specific surface area of packing

$C$ concentration of tracer in interstitial fluid

$C_{a} \quad$ surface concentration of tracer on solid surface

$C_{t}$ concentration of tracer in the fluid at the solid-liquid interface

d particle diameter -

$D_{A B} \quad$ binary diffusion coefficient

$E \quad$ dispersion coefficient

$f(t) \quad$ residence time probability density

$I \quad$ Nonuniformity index

$J_{E} \quad$ degree of segregation applied to exit plane

$J_{F} \quad$ degree of segregation based on flow filaments

$J_{\mathcal{S}} \quad$ degree of segregation for entire vessel

$k_{a} \quad$ adsorption rate constant

$k_{m} \quad$ mass transfer coefficient at particle surface

$k_{\text {eff }} \quad$ effective rate constant

$K$ equilibrium constant for adsorption

$L \quad$ length of bed in direction of flow 
$M_{E} \quad$ Index of micromixing for the exit plane

$M_{F} \quad$ Index of micromixing based on flow filaments

$n$ mass flux of adsorbing/desorbing specie

$P \quad$ rate parameter defined in text

$P e \quad$ Peclet number, $v L / E$

$Q \quad$ rate parameter defined in text

$R \quad$ capacity factor $=P / Q=a K / \epsilon$

$\mathrm{Re} \quad$ particle Reynols number, $\rho \nu d / \mu$

$s \quad$ Laplace transform variable

$S c \quad$ Schmidt number, $\mu / \rho D_{A B}$

$t$ time (s) or dimensionless time

$v \quad$ interstitial velocity

$V \quad$ Total fluid volume

$V_{0} \quad$ Total bed volume

$z \quad$ axial coordinate

Greek letters

$\alpha \quad$ age

$\alpha_{p} \quad$ mean age at a point or in a filament

$\delta(t)$ delta function

$\delta z \quad$ infinitesimal increment in the $\mathrm{z}$ direction

$\rho \quad$ Fluid density

$\epsilon \quad$ void fraction of packing $=V N_{o}$

$\sigma_{p} \quad$ standard deviation of age distribution at a point or for a single filament

$\mu_{i} \quad \mathrm{i}$ th. moments of residence time probability density function

$\mu \quad$ viscosity of interstitial fluid

$\phi(\alpha)$ age probability density function

\section{REFERENCES}

Danckwerts, P.V., 1958, The effect of incomplete mixing on homogeneous reactions.

Chem. Engng. Sci. 8, 93.

Gibbs, S. J. and Lightfoot, E.N., 1986, Scaling up gradient elution chromatography. Ind. Eng. Chem. Fundam. 25, 490.

Haughy, D.P. and Beveridge, G.S.G., 1966, Local voidage variation in a randomly packed bed of equal-sized spheres. Chem. Engng. Sci. 21, 905. 
Hejtamek, V. and Schneider, P., 1993, Axial dispersion under liquid-chromatography conditions. Chem. Engng. Sci. 48, 1163.

Lee, M.L., Yang, F.J., and Bartle, K.D., 1984, Open Tubular column gas chromatography. John Wiley and Sons, New York. (Chapter 3.)

Nauman, E.B. and Buffham, B.A., 1983, Mixing in contimuous flow systems, John Wiley and Sons, New York (Chapter 3).

Perkins, R.A., and Jones, M.C., 1989, Fiber-optic fluorescence array to study free convection in porous media. Rev. Sci. Instrum. 60, 3492.

Reid, R.C., Prausnitz, J.M., and Poling, B.E., 1987, The properties of gases and liquids, 4th. edition. McGraw-Hill, New York.

Wen, C.Y., and Fan, L.T., 1975, Models for flow systems and chemical reactors, Marcel Dekker, New York.

Zwietering, T.N., 1959, The degree of mixing in continuous flow systems. Chem. Engng. Sci. 11, 1.

\section{APPENDIX}

The corresponding general moments for the one-dimensional flow model with adsorption and dispersion may be obtained from the equilibrium adsorption moments, equations (15) (17), by multiplying by the following factors $r_{i}$ :

$r_{1}-1$

$r_{2}=1+R / Q$

$r_{3}=1+(R / P e)(2 R+R \cdot P e+P e+2) / Q+R / Q 2$.

Where $Q=k_{e f f} L / K \nu$. Thus, as the overall kinetic/mass transfer coefficient $k_{e f f}$ declines, the response broadens but also becomes more skewed. The equilibrium adsorption case is recovered as $Q$ approaches infinity. 


\section{LIST OF FIGURES}

Figure 1. Experimental system.

Figure 2. Plot showing typical individual probe responses

Figure 3. Second moment of tracer response as a function of the number of probes used in its calculation. Bed $8,3 \mathrm{~mm}$ spheres.

Figure 4. Overall response fit to a linear combination of two functions as given by equation (13). For first function, $\mathrm{a}=$ mean time; $\mathrm{b}=P e ; \mathrm{c}=$ weight. For second function, $\mathrm{d}=$ weight; $\mathrm{e}=$ mean time; $\mathrm{f}=P e$.

Figure 5. The ratio $M_{F}$ as a function of $P e$ for experimental results. The solid line is the theoretical result from the ideal dispersion model.

Figure 6. Plot of $M_{F}$ vs Re.

Figure 7. Plot of $M_{E}$ vs $P e$.

Figure 8. Nonuniformity index $I$ vs $P e$. The symbols have the same connotations as in Figure 5.

Figure 9. Particle Peclet $P e_{d}$ number from fits to tracer responses as a function of Particle Reynolds Number, Re. 


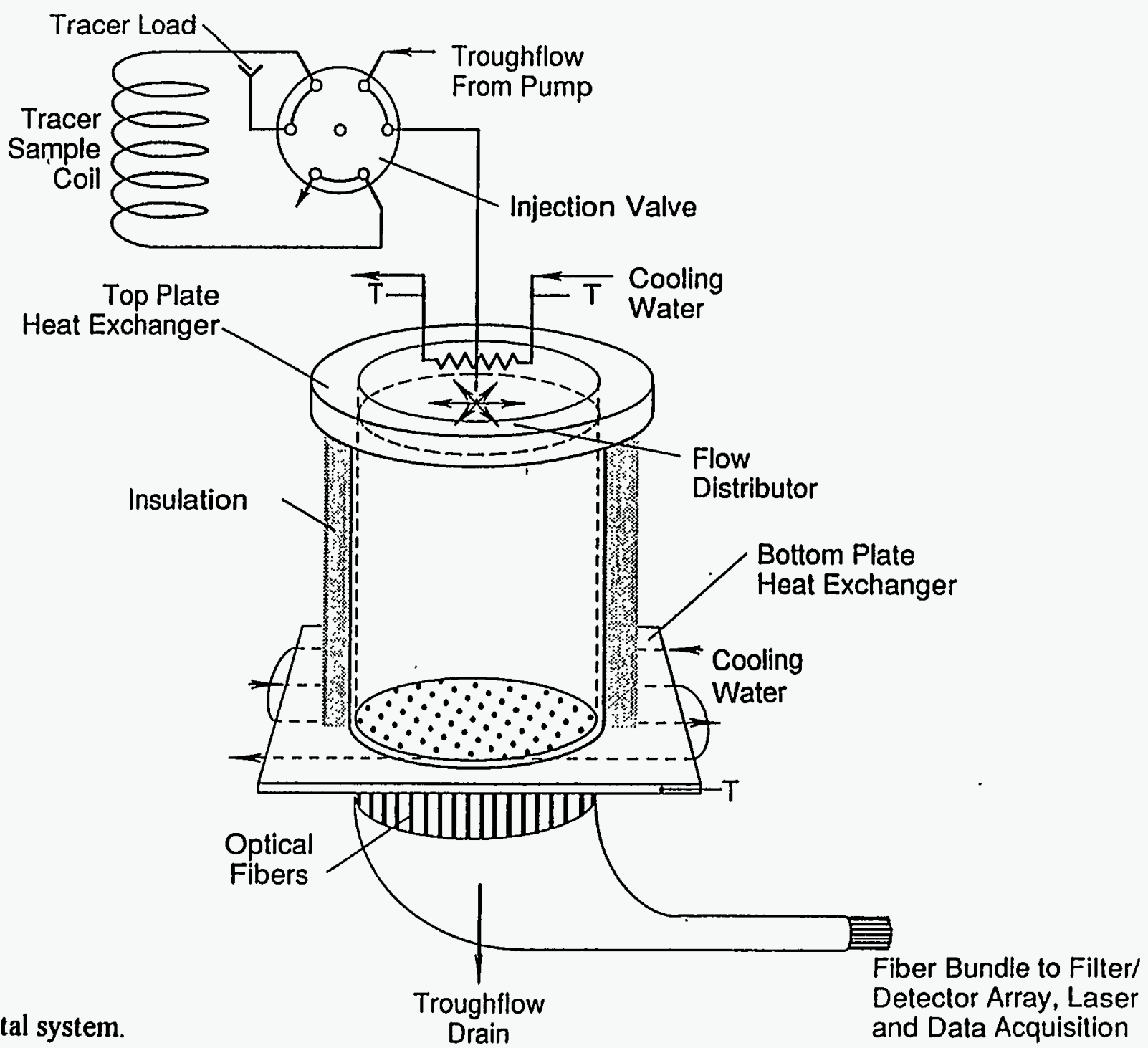




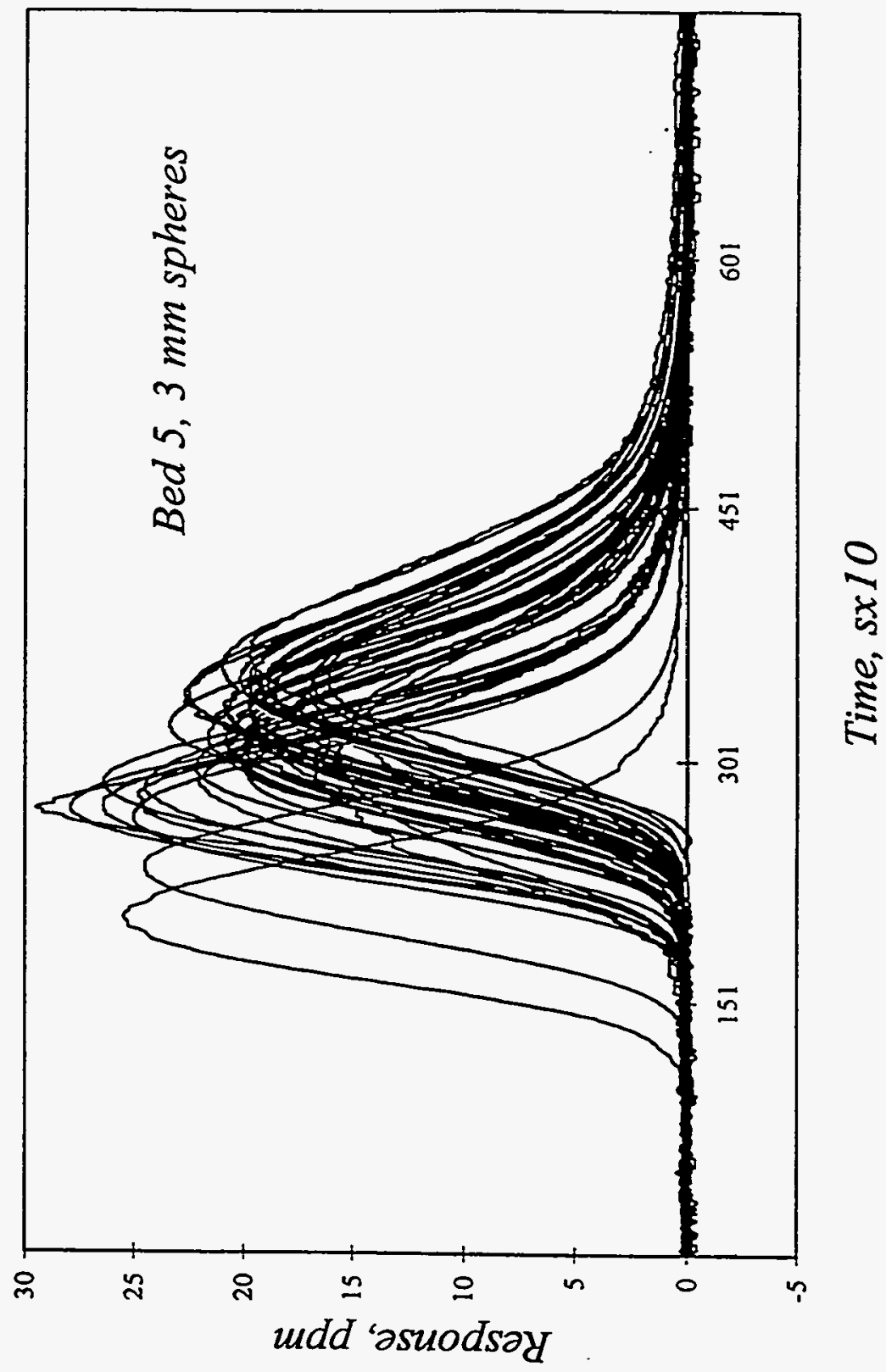

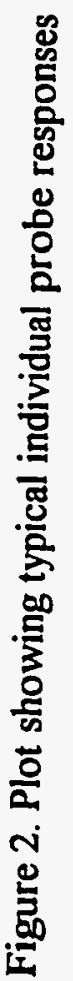




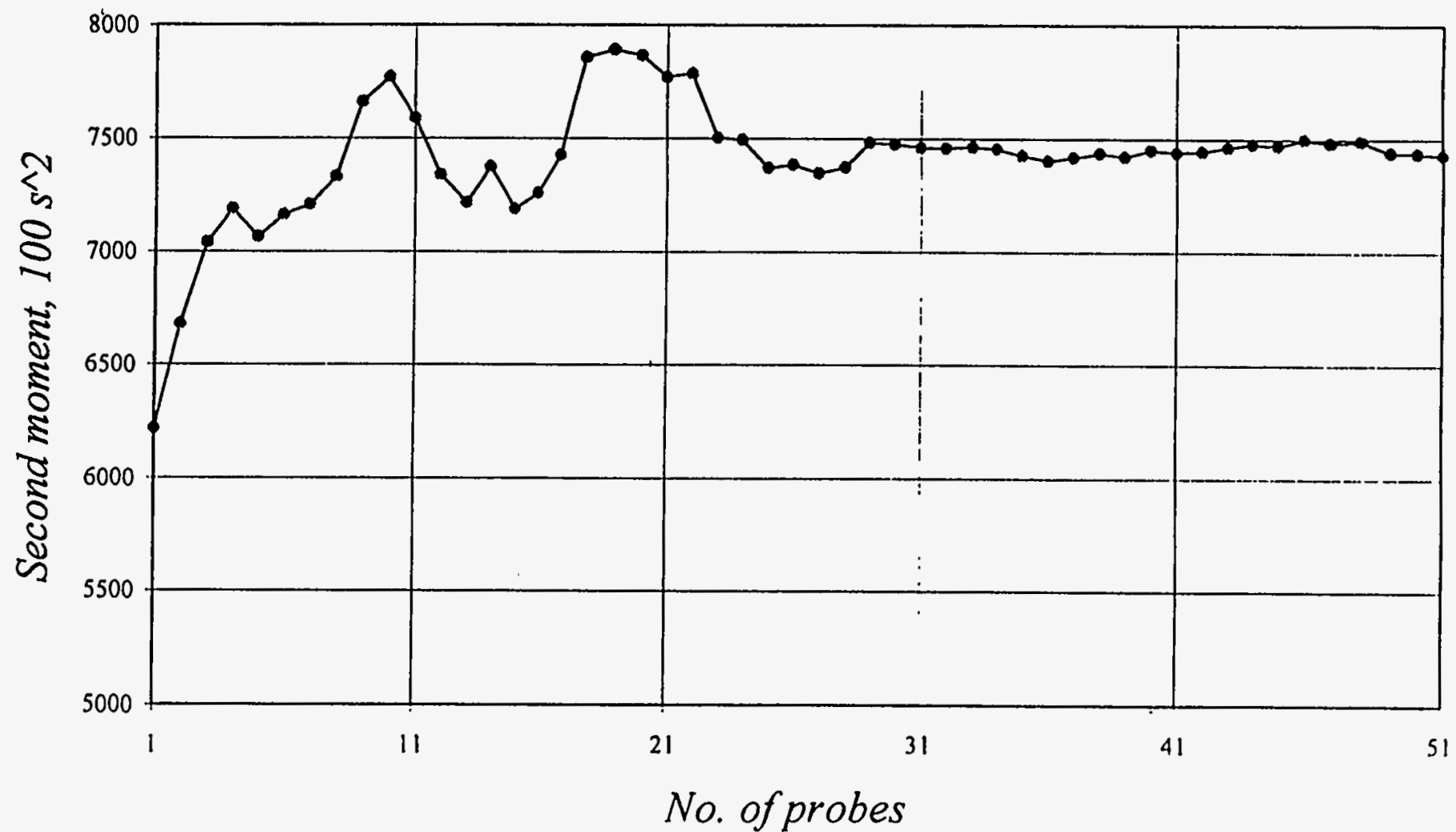

Figure 3. Second moment of tracer response as a function of the number of probes used in its calculation. Bed 8, $3 \mathrm{~mm}$ spheres. 


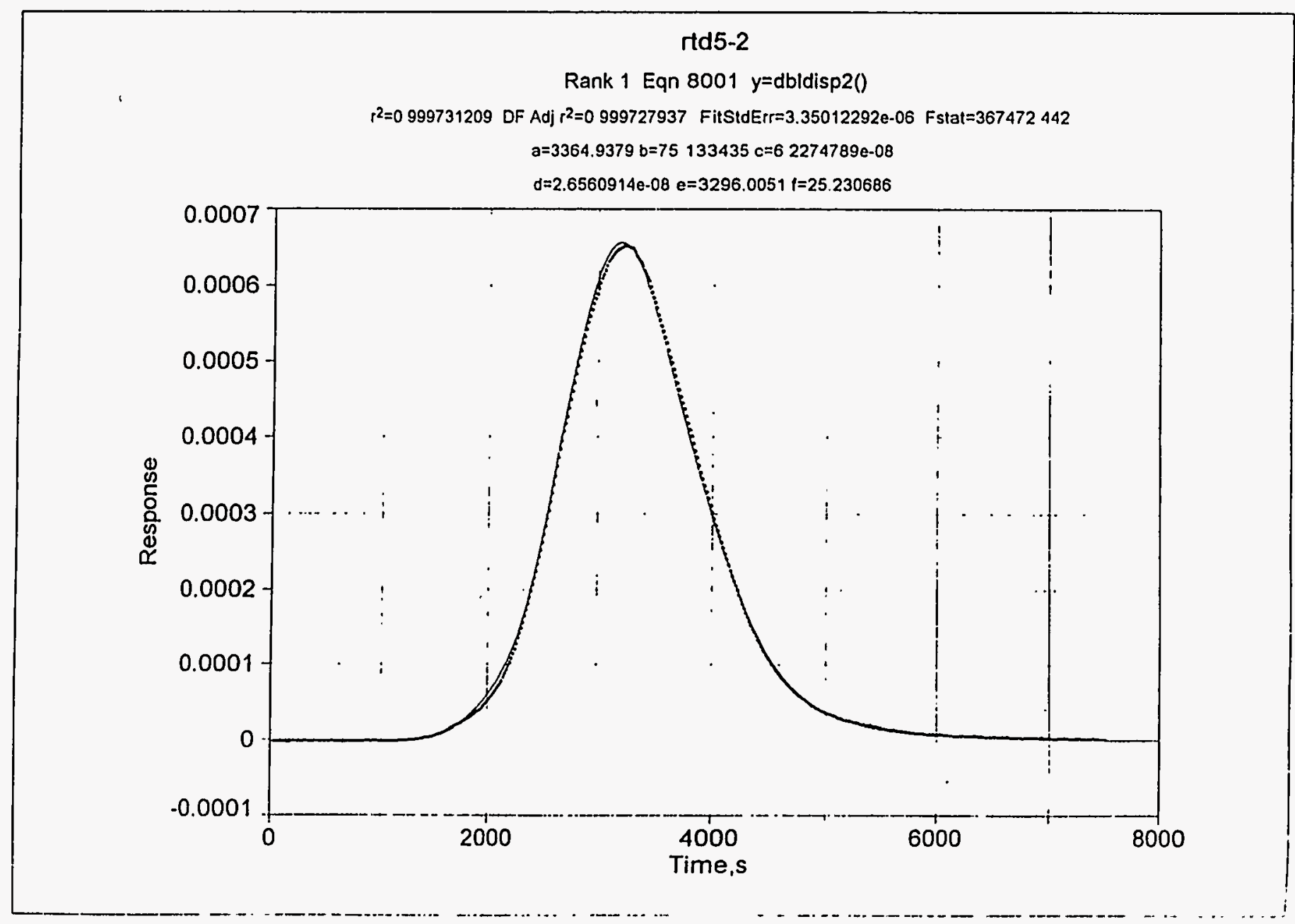

Figure 4. Overall response fit to a linear combination of two functions as given by equation (13). For first function, $a=$ mean time; $b=P e ; c=$ weight. For second function, $\mathrm{d}=$ weight; $\mathrm{e}=$ mean time; $\mathrm{f}=P e$. 


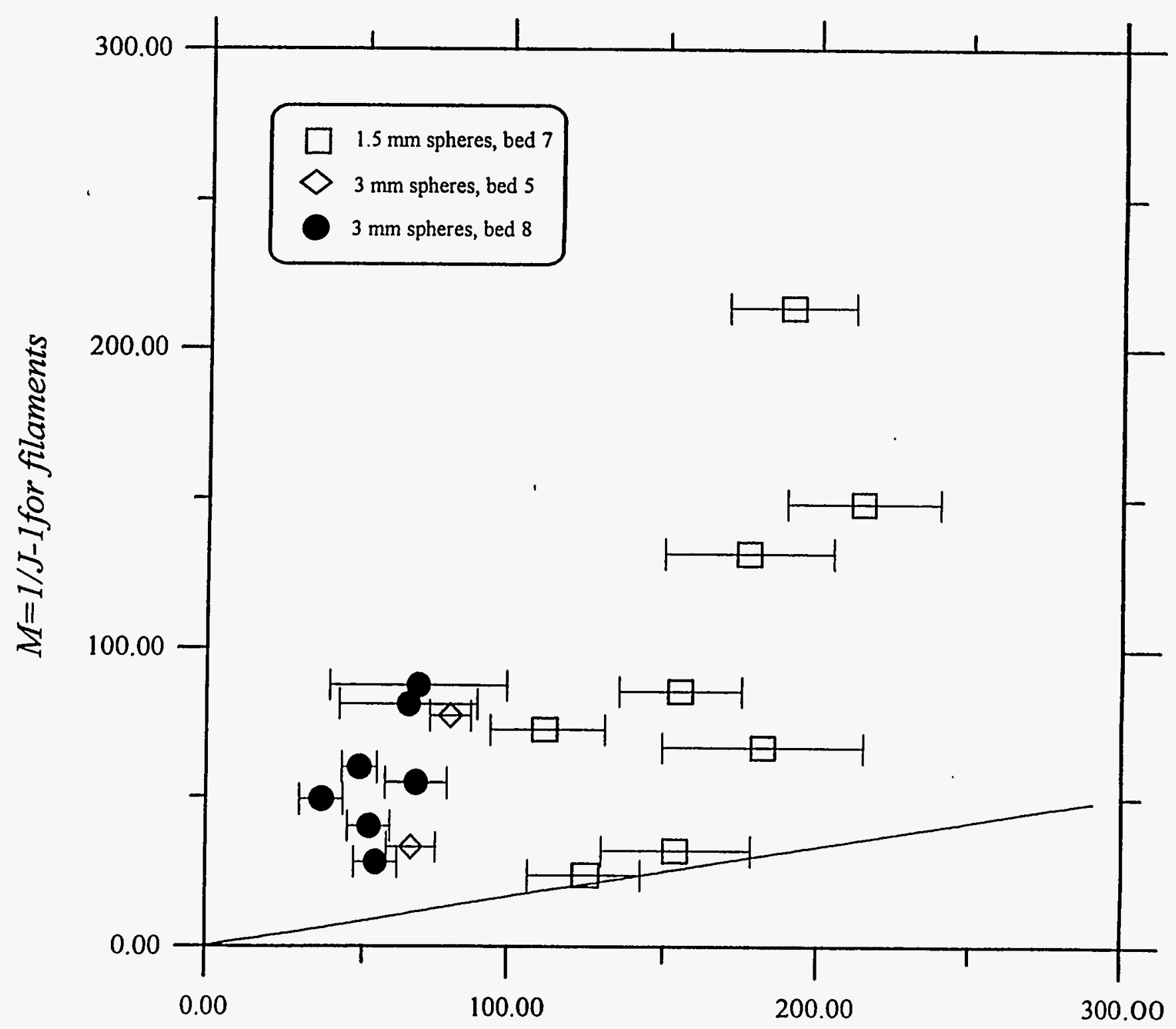

Figure 5. The ratio $M_{F}$ as a function of $P e$ for experimental results. The solid line is the theoretical result from the ideal dispersion model. 


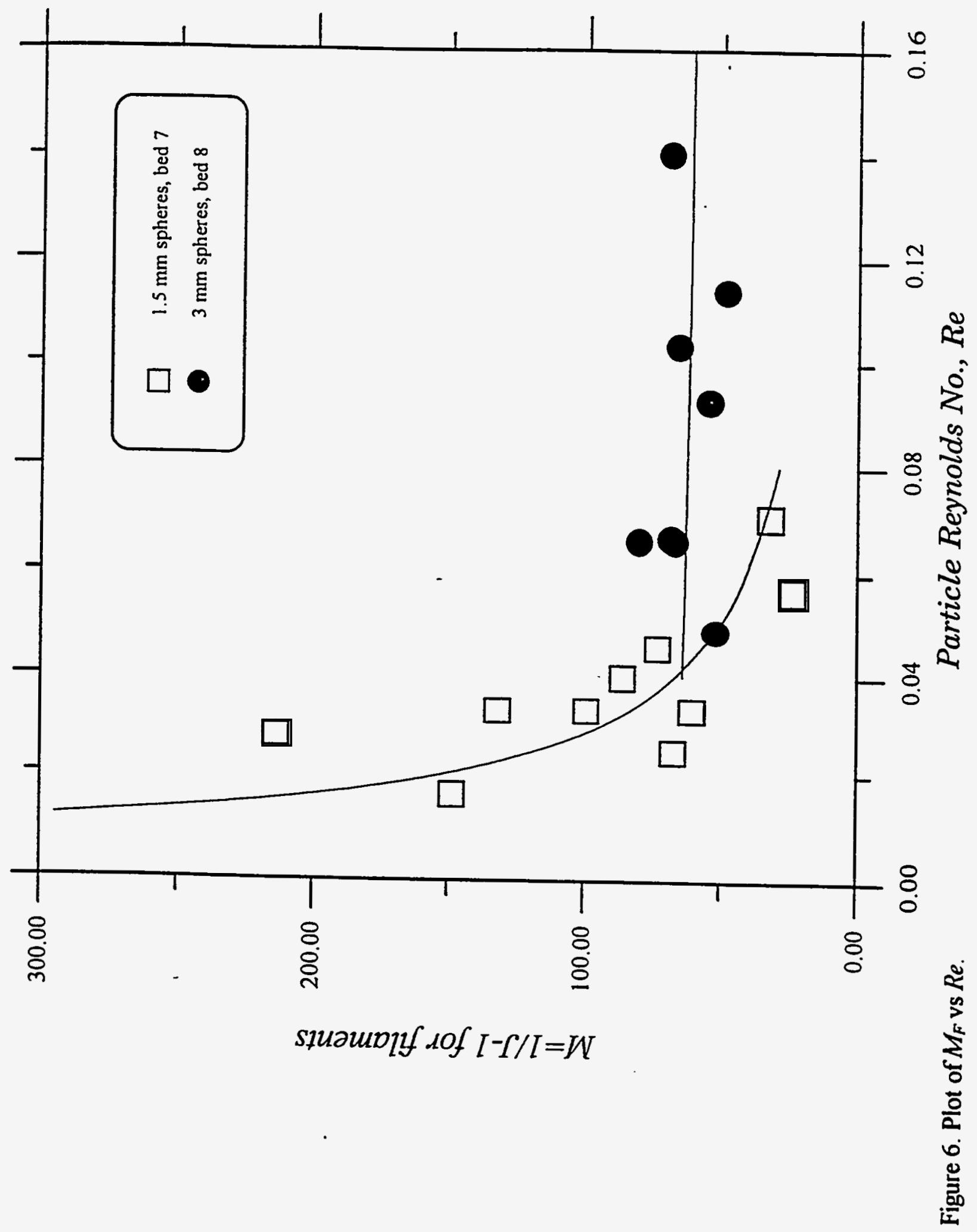




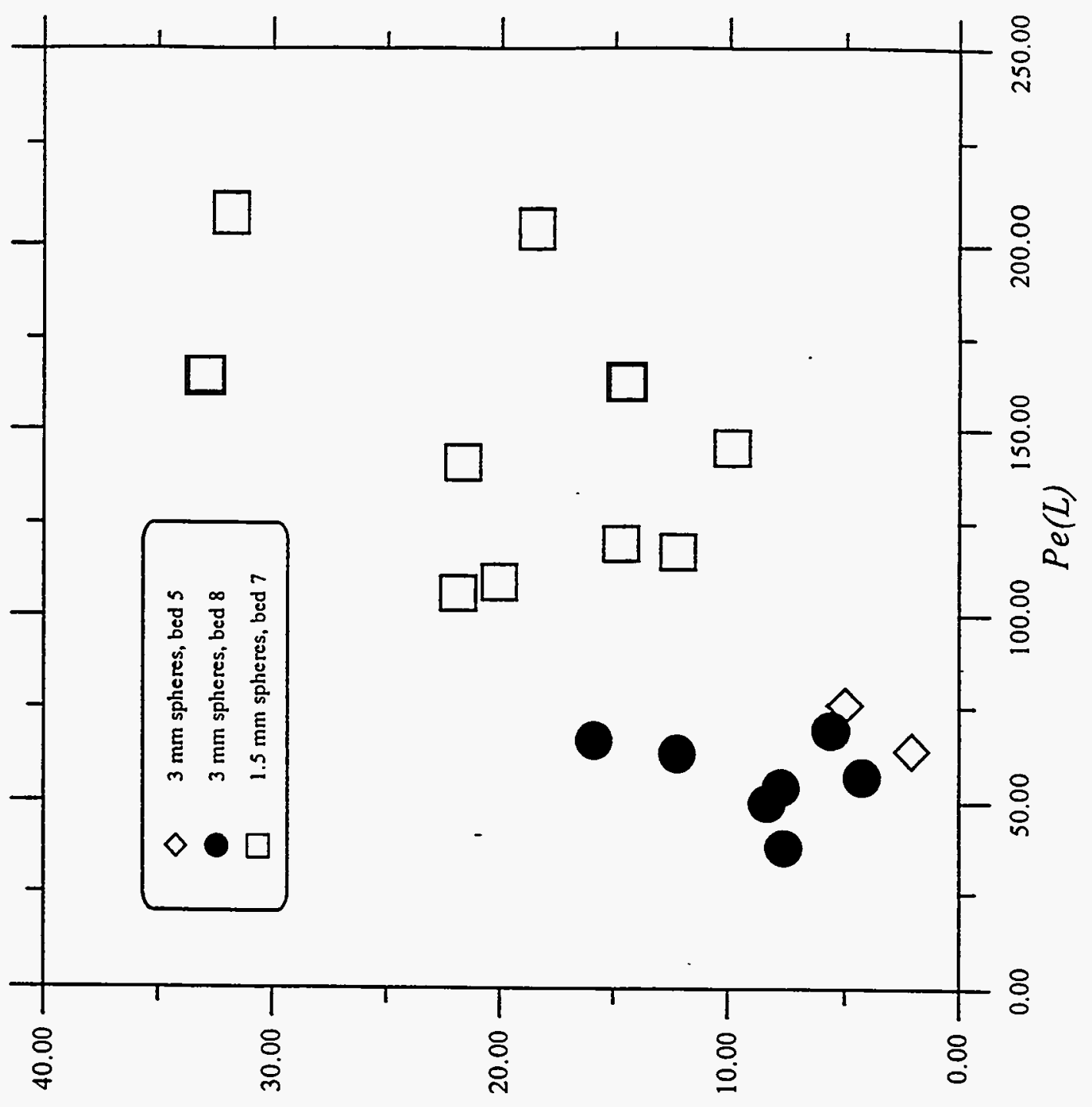

aupld 1!xa dof $l-\Gamma / I=W$

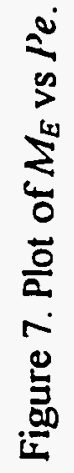




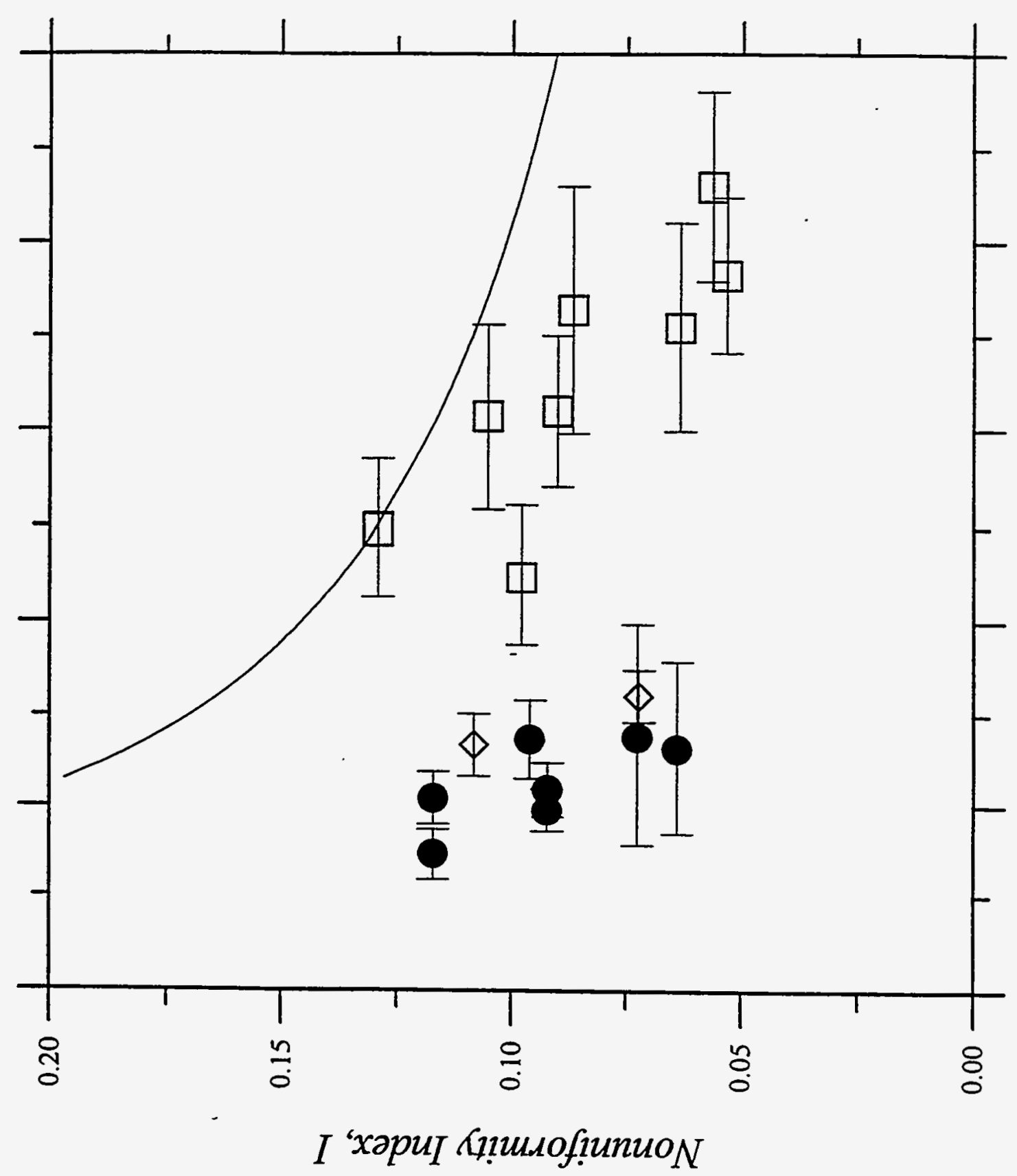

0
0
0
ำ

옹
옹

8
0
0
$\circ$

बे

용

.

ส

옹

.

용 


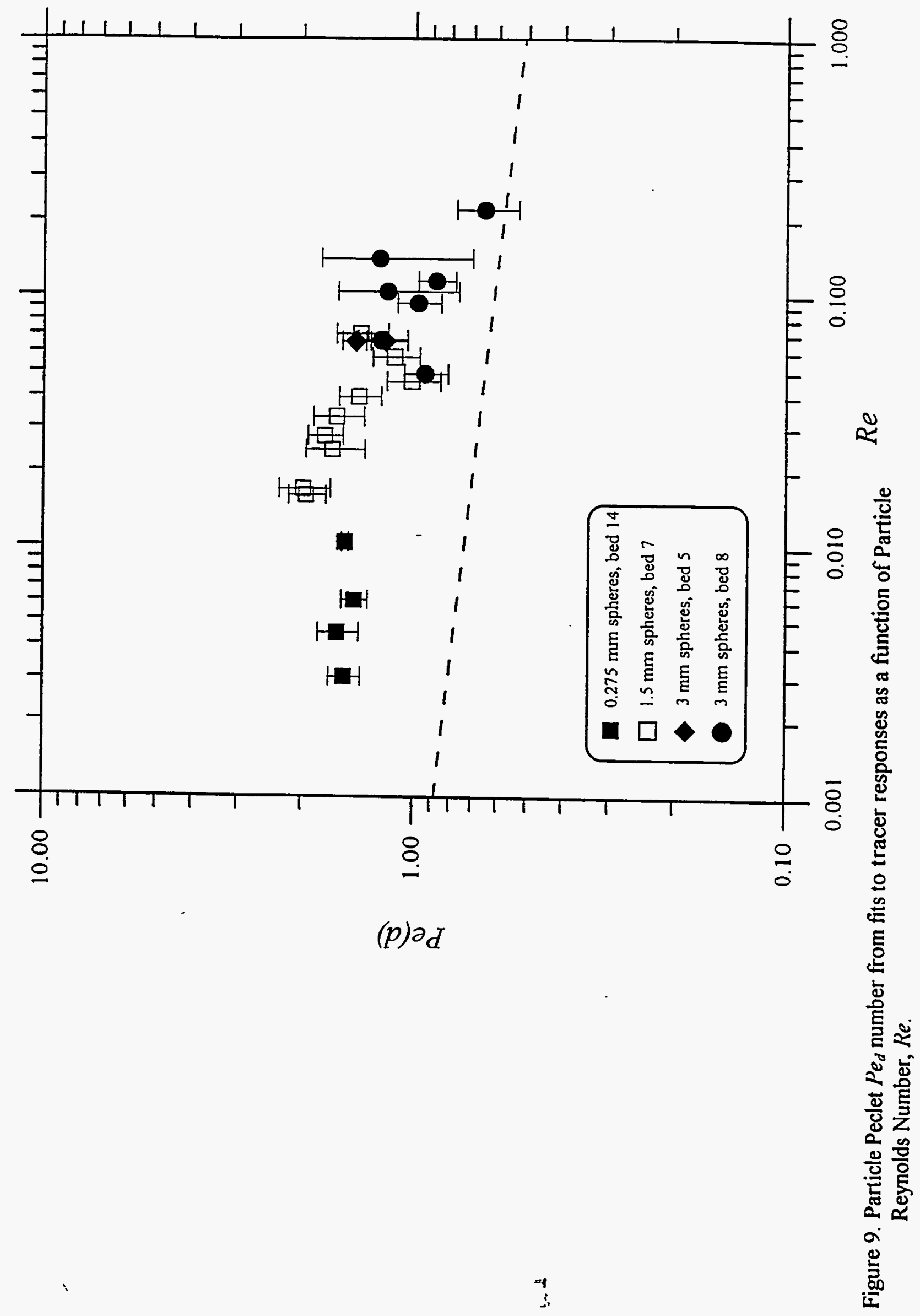

